\title{
Theoretical Solution Energy of Alkaline Earth Ions in Lanthanum Chromites
}

\author{
Hiroki Moriwake ${ }^{1}$, Isao Tanaka ${ }^{1}$, Kazuyoshi Tatsumi $^{1}$, Yukinori Koyama ${ }^{1}$, \\ Hirohiko Adachi ${ }^{1}$, Hisataka Yakabe ${ }^{2}$ and Isamu Yasuda ${ }^{2}$ \\ ${ }^{1}$ Department of Materials Science and Engineering, Kyoto University, Kyoto 606-8501 Japan \\ ${ }^{2}$ Fundamental Technology Research Laboratory, Tokyo Gas Co. Ltd., Tokyo 105-0023, Japan
}

\begin{abstract}
First principles calculations by a plane-wave basis pseudopotential method have been made for $\mathrm{Mg}$, $\mathrm{Ca}$, and $\mathrm{Sr}$-doped lanthanum chromites $\left(\mathrm{LaCrO}_{3}\right)$. A supercell composed of 40 atoms of a high temperature phase of $\mathrm{LaCrO}_{3}$ having a cubic-perovskite structure has been used. Solution energies of a neutral solute and a compensated solute by an oxygen vacancy were systematically computed. They were obtained for two kinds of cation sites and four thermodynamical conditions with different chemical potentials of constituent atoms. Mg shows lowest solution energy when it substitutes for the $\mathrm{Cr}$ ion. On the other hand, both $\mathrm{Ca}$ and $\mathrm{Sr}$ prefer to be located at La site. The charge neutral states are preferred by all of them. The results are consistent to experimental results regarding the site preference and the charge state.
\end{abstract}

(Received February 18, 2002; Accepted March 22, 2002)

Keywords: first principles calculations, solution energy, lanthanum chromites, neutral solute, compensated solute

\section{Introduction}

Alkaline earth (AE)-doped lanthanum chromites $\left(\mathrm{LaCrO}_{3}\right)$ have been used in solid oxide fuel cells (SOFC) as interconnectors that are required to show high electrical conductivity and stability at high temperatures within a wide range of oxygen partial pressure, $p_{\mathrm{O}_{2}}{ }^{1,2)}$ Recently application of these materials to new anode materials for direct methane oxidation has been contemplated. ${ }^{3)}$ They are expected to substitute conventional Ni/YSZ (ytteria stabilized zirconia) anodes that have been optimized for hydrogen fuel.

In $\mathrm{Mg}, \mathrm{Ca}$ or $\mathrm{Sr}$-doped $\mathrm{LaCrO}_{3}$, holes are introduced by the substitution of either trivalent $\mathrm{La}$ or $\mathrm{Cr}$ by divalent $\mathrm{AE}$ when $p_{\mathrm{O}_{2}}$ is high, which brings about high $p$-type conductivity. In reducing atmosphere, oxygen contents decrease by the formation of oxygen vacancies in order to compensate the charge of the solute atoms. It has been shown that the formation of oxygen vacancies is not detectable when $p_{\mathrm{O}_{2}}$ is higher than approximately $10^{-5} \mathrm{~Pa}$ at $1000^{\circ} \mathrm{C}$. Electrical conductivity decreases in reducing atmosphere when $p_{\mathrm{O}_{2}}$ is lower than the critical value. At the same time, crystalline lattice expands with the decrease of the oxygen content. ${ }^{4,5}$ ) The volume expansion generates residual stress in the interconnectors of SOFC under operating cycles, which eventually leads to macroscopic fracture of the components. It is therefore essential to have detailed knowledge on oxygen vacancies in $\mathrm{LaCrO}_{3}$.

Despite the importance, fundamental knowledge of defects in $\mathrm{LaCrO}_{3}$ is limited. Energetics of solutes and/or vacancies have not been investigated thus far by experiments. No theoretical calculations to estimate them have been undertaken. In this study, we perform first principles calculations of $\mathrm{Mg}, \mathrm{Ca}$ or Sr-doped $\mathrm{LaCrO}_{3}$ in order to evaluate the heats of solution for uncompensated/compensated solutes.

$\mathrm{LaCrO}_{3}$ can be categorized in strongly correlated systems in which one-electron approximations such as LDA (local density approximation) fail to reproduce properties like bandgap and magnetism. ${ }^{6}$ If we are interested in these proper- ties, Coulomb interactions among $d$-electrons should be taken into account explicitly beyond the one-electron approximation for example using the $U$-parameter that measures effective Coulomb interactions among $d$-electrons. However, such approach can be applied only to simple systems because of computational complexity. Calculations of defects with a large supercell require great care. In the present study we performed one electron calculations in an ordinary manner. Comparison with experimental data has revealed that the calculations by the one-electron approximations are very useful.

\section{Computational Procedure}

$\mathrm{LaCrO}_{3}$ shows a number of phase transformations at elevated temperatures. Recent convergent-beam electron diffraction experiment ${ }^{7)}$ has reported that the phase stable at room temperature has orthorhombic symmetry $($ Pbnm) with $a=$ $0.5479, b=0.5516$ and $c=0.7766 \mathrm{~nm}$. Above $1650^{\circ} \mathrm{C}$, a transformation to a cubic perovskite phase has been known to take place. In the present study, we adopted the cubic phase for computational economy.

All calculations were performed within the generalized gradient approximation $(\mathrm{GGA})^{8)}$ of the density functional theory, using a plane-wave pseudopotential method. ${ }^{9,10)}$ Spinpolarization is taken into consideration when $\mathrm{Cr}$ atoms were included in the supercell. Calculations for solutes and vacancies were made using the super-cell composed of 40 atoms that is 8 times greater than the primitive cell. Ultrasoft pseudopotentials $^{11)}$ were employed with a plane-wave cutoff energy of $400 \mathrm{eV}$. The convergence of the solution energies with respect to the cutoff energy up to $800 \mathrm{eV}$ was better than $0.01 \mathrm{eV}$. Numerical integration was carried out using $8 \mathrm{k}$ points in the whole Brillouin zone of the supercell. The convergence with respect to the number of $\mathbf{k}$-points was better than that of the cut-off energy. For simplicity, spins of neighboring $\mathrm{Cr}$ atoms were assumed to be parallel for $\mathrm{LaCrO}_{3}$ and $\mathrm{Cr}_{2} \mathrm{O}_{3}$.

Atomic arrangements around solutes and vacancies were 
optimized allowing relaxation of the first and second nearest neighbors. The relaxation procedures were truncated when all the residual forces for the relaxed atoms were smaller than $1 \mathrm{eV} / \mathrm{nm}$ We also carried out calculations of a number of reference materials, i.e., six kinds of elementary substances, $\mathrm{Cr}_{2} \mathrm{O}_{3}(R \overline{3} c), \mathrm{La}_{2} \mathrm{O}_{3}(P \overline{3} m 1)$ and three kinds of AE monooxides $(F m \overline{3} m)$. Total energies of the reference materials were obtained after their structures were optimized by the same computational method for consistency.

Formation energies of defects in compounds depend on chemical potentials of constituent atoms in general. For example, the solution energy of $\mathrm{AE}$ in $\mathrm{LaCrO}_{3}$ at the La site can be given using the following equation when it is neutral $(q=0)$ :

$$
\begin{aligned}
E^{\mathrm{S}}\left[\mathrm{AE}_{\mathrm{La}}, q=0\right]= & E_{\mathrm{T}}\left(\mathrm{La}_{n-1} \mathrm{AECr}_{n} \mathrm{O}_{3 n}\right)-E_{\mathrm{T}}\left(\mathrm{La}_{n} \mathrm{Cr}_{n} \mathrm{O}_{3 n}\right) \\
& +\mu_{\mathrm{La}}-\mu_{\mathrm{AE}}
\end{aligned}
$$

where $E_{\mathrm{T}}$ is the total energy of the supercell, and $\mu_{\mathrm{X}}$ is the atomic chemical potential of X. $n$ is the number of formula units included in the supercell, which was 8 in this work.

When a hole associated with an AE solute is compensated by the formation of oxygen vacancy, the solution energy can be calculated using a supercell of $\mathrm{La}_{n-2} \mathrm{AE}_{2} \mathrm{Cr}_{n} \mathrm{O}_{3 n-1}$ with neutral charge in principle. However, calculations with three defects require a large supercell in order to avoid artificial interactions among defects. For computational simplicity, we have adopted an alternative way. The solution energy of a compensated solute per the set of the solute and a half of the oxygen vacancy $\left(\mathrm{V}_{\mathrm{O}}\right)$ was evaluated by the sum of $E\left[\mathrm{AE}_{\mathrm{La}}\right.$, $q=-1]$ and a half of $E\left[\mathrm{~V}_{\mathrm{O}}, q=+2\right]$ by two separate calculations using the following equation:

$$
\begin{aligned}
E^{\mathrm{S}}\left[\mathrm{AE}_{\mathrm{La}}+1 / 2 \mathrm{~V}_{\mathrm{O}}\right]= & E_{\mathrm{T}}\left(\mathrm{La}_{n-1} \mathrm{AECr}_{n} \mathrm{O}_{3 n}, q=-1\right) \\
& +1 / 2 E_{\mathrm{T}}\left(\mathrm{La}_{n} \mathrm{Cr}_{n} \mathrm{O}_{3 n-1}, q=+2\right) \\
& -3 / 2 E_{\mathrm{T}}\left(\mathrm{La}_{n} \mathrm{Cr}_{n} \mathrm{O}_{3 n}, q=0\right) \\
& +\mu_{\mathrm{La}}-\mu_{\mathrm{AE}}+1 / 2 \mu_{\mathrm{O}}
\end{aligned}
$$

The non-zero $(q \neq 0)$ charge of supercells was neutralized by a standard method using jellium background. The use of eq. (2) corresponds to the case that the AE solute and the oxygen vacancy are located without making any interactions but maintain the same Fermi energy.

\section{Results and Discussion}

\subsection{Formation energy of compounds}

In order to know the accuracy of the present calculations, we firstly examined the formation energies of perfect compounds. The formation energies of many binary oxides have been reported in literature. The theoretical value that corresponds to the experimental formation energy can be obtained using three theoretical values as

$$
\begin{aligned}
\Delta E\left[\mathrm{M}_{a} \mathrm{O}_{b}\right]= & E_{\mathrm{T}}\left[\mathrm{M}_{a} \mathrm{O}_{b}\right]-\left(a \times E_{\mathrm{T}}[\mathrm{M}(\text { solid })]\right. \\
& \left.+b / 2 \times E_{\mathrm{T}}\left[\mathrm{O}_{2}(\text { solid })\right]\right)
\end{aligned}
$$

when we neglect any temperature effects. Experimental data use $\mathrm{O}_{2}$ (gas) as the standard not with the $\mathrm{O}_{2}$ (solid). We have used $\mathrm{O}_{2}$ (solid) with the space group of $C 2 / m$ as the standard.
However, the difference in $E_{\mathrm{T}}$ between the gas and the solid was found to be only $0.04 \mathrm{eV} /$ atom. Theoretical formation energies, $\Delta E\left[\mathrm{M}_{a} \mathrm{O}_{b}\right]$, are listed in Table 1 together with available experimental data. ${ }^{12)}$ It can be found that present theoretical calculations underestimate the energy for all of five compounds by 7 to $22 \%$. The errors by a few $10 \%$ should therefore be taken into consideration when we use the absolute values of the energies. The underestimation may be ascribed to the neglect of temperature effects. However, the systematic trend in errors suggests that relative errors among different defect configurations are much smaller.

\subsection{Site preference and energy of solution}

It has been widely accepted that $\mathrm{Mg}$ atoms occupy $\mathrm{Cr}$ sites in $\mathrm{LaCrO}_{3}$. On the other hand, $\mathrm{Sr}$ and $\mathrm{Ca}$ atoms are known to occupy La sites. The site preferences are quite natural from the viewpoint of ionic size. The ionic radius ${ }^{13)}$ of $\mathrm{Mg}, r_{\mathrm{Mg}^{2+}}$ is $86 \mathrm{pm}$, which is much closer to $r_{\mathrm{Cr}^{3+}}=76 \mathrm{pm}$ than $r_{\mathrm{La}^{3+}}=$ $117 \mathrm{pm}$. On the other hand, $r_{\mathrm{Ca}^{2+}}=114 \mathrm{pm}$ and $r_{\mathrm{Sr}^{2+}}=$ $132 \mathrm{pm}$ are closer to $r_{\mathrm{La}^{3+}}$ than $r_{\mathrm{Cr}^{3+}}$. The use of such a rigid sphere model may not always be valid, although it provides a good insight.

In order to evaluate the solution energy as given by eqs. (1) and (2), we have to define all chemical potentials of constituent atoms. Four kinds of conditions as shown in a schematic phase diagram of Fig. 1 have been adopted. Preferable phase compositions were determined using theoretical formation energies as shown in Table 1. For example, the composition $\mathrm{La}_{0.4} \mathrm{Cr}_{0.4} \mathrm{O}_{0.2}$ can be composed of either $1 \mathrm{LaCrO}_{3}+5 \mathrm{La}+5 \mathrm{Cr}$ or $1 \mathrm{La}_{2} \mathrm{O}_{3}+4 \mathrm{La}+6 \mathrm{Cr}$. The latter model is found to show lower energy by $0.22 \mathrm{eV} /$ atom. The composition $\mathrm{La}_{0.25} \mathrm{Cr}_{0.25} \mathrm{O}_{0.5}$ can be composed of either

Table 1 Theoretical formation energy of six compounds in comparison with experimental values.

\begin{tabular}{lccc}
\hline & \multicolumn{3}{c}{ Formation energy/eV } \\
\cline { 2 - 4 } & Theory & Experiment & Theo/exp \\
\hline $\mathrm{La}_{2} \mathrm{O}_{3}$ & -15.93 & -18.59 & 0.86 \\
$\mathrm{Cr}_{2} \mathrm{O}_{3}$ & -9.14 & -11.76 & 0.78 \\
$\mathrm{MgO}$ & -5.48 & -6.24 & 0.88 \\
$\mathrm{CaO}$ & -6.13 & -6.58 & 0.93 \\
$\mathrm{SrO}$ & -5.69 & -6.14 & 0.93 \\
$\mathrm{LaCrO}_{3}$ & -12.56 & - & - \\
\hline
\end{tabular}

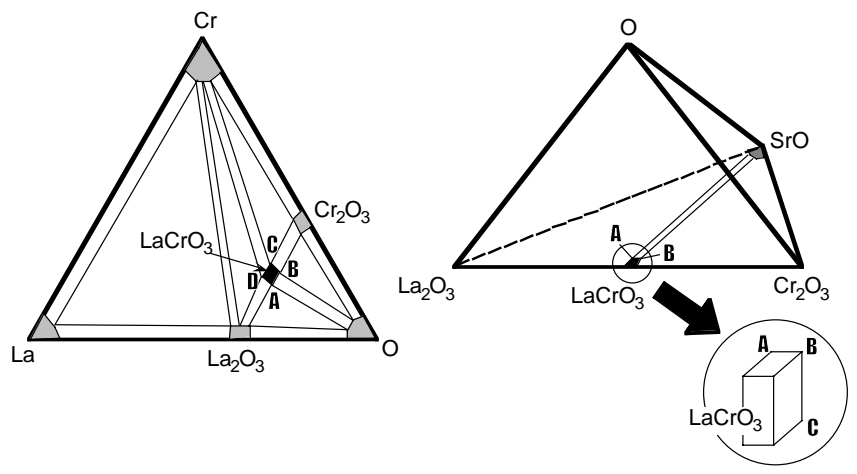

Fig. 1 Schematic phase diagrams for the $\mathrm{LaCrO}_{3}-\mathrm{AEO}$ system. A to D denote positions on the phase boundary of $\mathrm{LaCrO}_{3}$. 


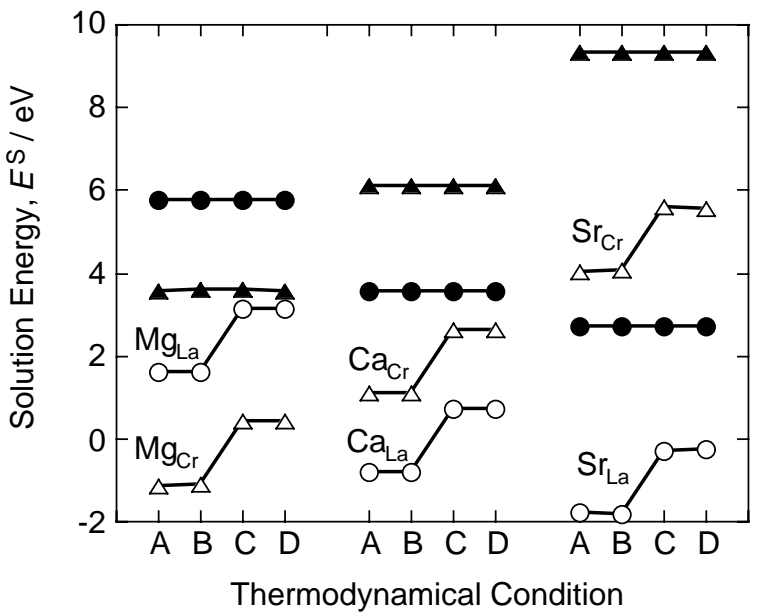

Fig. 2 Theoretical solution energy of $\mathrm{AE}$ atoms in $\mathrm{LaCrO}_{3}$. In this figure, circles show solution energy of substitution for $\mathrm{La}$ ion in $\mathrm{LaCrO}_{3}$, triangles show substitution for $\mathrm{Cr}$ ion in $\mathrm{LaCrO}_{3}$ respectively. Open circles and triangles denote the energy for uncompensated solutes (neutral charge). Closed marks show compensated solutes (compensated by $\mathrm{O}$ vacancy).

$2 \mathrm{LaCrO}_{3}+1 \mathrm{La}+1 \mathrm{Cr}$ or $1 \mathrm{LaCrO}_{3}+1 \mathrm{La}_{2} \mathrm{O}_{3}+2 \mathrm{Cr}$. The latter model shows $0.28 \mathrm{eV} /$ atom lower in energy. The phase diagram shown in Fig. 1 has been constructed on the basis of such information.

Figure 2 shows the solution energies calculated for four thermodynamical conditions denoted by A, B, C and D in Fig. 1. Presence of the AE monooxide was assumed for all cases. In other words, $\mu_{\mathrm{AE}}$ was always taken to be equal to $E_{\mathrm{T}}[\mathrm{AEO}]-\mu_{\mathrm{O}}$. The positions of four conditions in a pseudoquaternary diagram are also shown in Fig. 1. Four conditions are located on the surface of the $\mathrm{LaCrO}_{3}$ region facing the AEO region in the diagram.

As can be seen in Fig. 2, Mg shows lowest solution energy when it substitutes for the $\mathrm{Cr}$ ion and neutral in charge. On the other hand, both $\mathrm{Ca}$ and $\mathrm{Sr}$ prefer to be located at the La site. They also prefer the neutral charge-state. All these results are consistent to experimental results regarding the site preference. The experimental fact that all of them act as $p$-type dopants is also well reproduced by the calculation, because the solution energies of the charge-compensated defects are much higher than those of the neutral solutes. When the condition is changed from $\mathrm{B}$ to $\mathrm{C}, \mu_{\mathrm{O}}$ changes by $-3.0 \mathrm{eV}$, which corresponds to the difference in oxygen partial pressure by $5 \times 10^{-25}$ at $1273 \mathrm{~K}$. The solution energy of the neutral solute increases with the decrease of the $\mu_{\mathrm{O}}$, which is quite natural. The only problem we can see is the absolute values of theoretical solution energies. Oxygen vacancies are known to form gradually with the decrease of the oxygen partial pressure in the reducing atmosphere in the AE-doped $\mathrm{LaCrO}_{3}$. According to the present calculation, however, the difference in the solution energy between the neutral and the compensated dopants maybe too large even at conditions C and D to form any notable oxygen vacancies. The reason for the discrepancy is unclear at the present moment. However, the overestimation of the difference could be partly ascribed to the use of eq. (2) for the compensated solutes. There may be some binding energy between the doped AE and the oxygen vacancy, which decreases the solution energy of the compen- sated solutes. Another source of the discrepancy may be due to correlation effects caused by interactions among multiple $3 d$-electrons. They were neglected in the one-electron theory. The magnitude of the correlation effects may be dependent on the number of $d$-electrons per a $\mathrm{Cr}$ atom. Since the solution energy of the compensated defects were calculated using the charged supercell having different number of $d$-electrons as compared to the neutral supercell, the magnitude of the correlation effects for two models should be different in principle. There may be a systematic error between two energies evaluated by the two models because of the correlation effect.

Despite these problems, it should be emphasized that the present calculations by the one-electron theory can reproduce site preferences of solutes qualitatively. Combining experimental efforts and this type of calculations should provide good insights into materials science issues of this kind of compounds.

\section{Conclusion}

First principles calculations by a plane-wave basis pseudopotential method have been made for $\mathrm{Mg}, \mathrm{Ca}$, and $\mathrm{Sr}$-doped $\mathrm{LaCrO}_{3}$ within GGA. A supercell composed of 40 atoms of a high temperature phase $\mathrm{LaCrO}_{3}$ having cubic-perovskite structure has been used. Spin-polarization was taken into account for Cr. Solution energies of a neutral solute and a compensated solute by a half of an oxygen vacancy were obtained for two cation sites and four thermodynamical conditions with different chemical potentials of constituent atoms. $\mathrm{Mg}$ shows lowest solution energy when it occupies the $\mathrm{Cr}$ site. On the other hand, both $\mathrm{Ca}$ and $\mathrm{Sr}$ prefer to be located at the La site. The charge neutral states are preferred by all of them. The results are consistent to experimental results regarding the site preference. The fact that these solutes act as effective $p$-type dopants can also be well explained by the calculation. A possible problem due to the neglect of the vacancy-solute interactions in the compensated model and strong correlations among $d$-electrons have been pointed out on the evaluation of the difference in the solution energies of the neutral and the compensated solutes.

\section{Acknowledgements}

This work was supported by the Grant-in-Aid for priority area (No. 751) from MEXT, Japan.

\section{REFERENCES}

1) J. Mizusaki, S. Yamauchi, K. Fueki and A. Ishikawa: Solid State Ionics 12 (1984) 119-124.

2) B. K. Flandermeyer, M. M. Nasrallah, A. K. Agarwal and H. U. Anderson: J. Am. Ceram. Soc. 67 (1984) 195-198.

3) P. Vernoux, E. Djurado and M. Guillodo: J. Am. Ceram. Soc., 84 (2001) 2289-2295.

4) W. Schafer and R. Schmidberger: High Tech Ceramics, ed. by P. Vincenzini (Elsevier, Amsterdam, 1987) pp. 1737-1742.

5) I. Yasuda and M. Hishinuma: Electrochemistry 68 (2000) 526-530.

6) I. Solovyev, N. Hamada and K. Terakura: Phys. Rev. B 53 (1996) 71587170 .

7) T. Hashimoto, K. Takagi, K. Tsuda, M. Tanaka, K. Yoshida, H. Tagawa and M. Dokiya: J. Electrochem. Soc. 147 (2000) 4408-4410.

8) J. P. Perdew, J. A. Chevary, S. H. Vosko, K. A. Jackson, M. R. Pederson, D. J. Singh and C. Fiolhais: Phys. Rev. B 46 (1992) 6671-6687. 
9) V. Milman, B. Winkler, J. A. White, C. J. Pickard, M. C. Payne, E. V. Akhmatskaya and R. H. Nobes: Int. J. Quant. Chem. 77 (2000) 895910.

10) The CASTEP program code was used in the present study. [Accelrys Inc., San Diego, CA.]

11) D. Vanderbilt: Phys. Rev. B 41 (1990) 7892-7895.

12) W. G. Mallard and P. J. Linstrom, Eds., NIST Chemistry Web-
Book, NIST Standard Reference Database Number 69, February 2000, National Institute of Standards and Technology, Gaithersburg MD (http://webbook.nist.gov).

13) Ionic radii for 6-fold coordinated cations were obtained from the following reference: R. D. Shannon: Acta Crystallogr. A32 (1976) 751767. 